\title{
Roles of Nucleus Accumbens Core and Shell in Incentive-Cue Responding and Behavioral Inhibition
}

\author{
Frederic Ambroggi, ${ }^{1,2,3^{*}}$ Ali Ghazizadeh, ${ }^{1,2,3,6^{*}}$ Saleem M. Nicola, ${ }^{4,5}$ and Howard L. Fields ${ }^{1,2,3}$ \\ ${ }^{1}$ Ernest Gallo Clinic and Research Center, ${ }^{2}$ Wheeler Center for the Neurobiology of Addiction, and ${ }^{3}$ Department of Neurology, University of California, San \\ Francisco, Emeryville, California 94608, and ${ }^{4}$ Department of Psychiatry and Behavioral Science and ${ }^{5}$ Dominick P. Purpura Department of Neuroscience, \\ Albert Einstein College of Medicine, Bronx, New York 10461, and 'Joint Bioengineering Program, University of California, San Francisco/University of \\ California, Berkeley, in San Francisco, California 94158-2230 and Berkeley, California 94720-1762
}

The nucleus accumbens (NAc) is involved in many reward-related behaviors. The NAc has two major components, the core and the shell. These two areas have different inputs and outputs, suggesting that they contribute differentially to goal-directed behaviors. Using a discriminative stimulus (DS) task in rats and inactivating the NAc by blocking excitatory inputs with glutamate antagonists, we dissociated core and shell contributions to task performance. NAc core but not shell inactivation decreased responding to a reward-predictive cue. In contrast, inactivation of either subregion induced a general behavioral disinhibition. This reveals that the NAc actively suppresses actions inappropriate to the DS task. Importantly, selective inactivation of the shell but not core significantly increased responding to the nonrewarded cue. To determine whether the different contributions of the NAc core and shell depend on the information encoded in their constituent neurons, we performed electrophysiological recording in rats performing the DS task. Although there was no firing pattern unique to either core or shell, the reward-predictive cue elicited more frequent and larger magnitude responses in the NAc core than in the shell. Conversely, more NAc shell neurons selectively responded to the nonrewarded stimulus. These quantitative differences might account for the different behavioral patterns that require either core or shell. Neurons with similar firing patterns could also have different effects on behavior due to their distinct projection targets.

\section{Introduction}

Nucleus accumbens (NAc) neurons contribute to behaviors elicited by reward-predictive sensory cues (Cardinal et al., 2002; Nicola, 2007; Humphries and Prescott, 2010). Lesions or pharmacological manipulation of the NAc impair the ability of conditioned stimuli (CS) to promote operant responding for drugs of abuse or natural rewards (Cardinal et al., 2002; Di Ciano et al., 2008; Floresco et al., 2008). However, reversible inactivation of the NAc failed to reduce cue responding in a discriminative stimulus (DS) task and instead increased unreinforced lever pressing (Yun et al., 2004a). In contrast to general inactivation of the NAc, blockade of dopamine receptors in the core strongly reduces incentive-cue responding (Ambroggi et al., 2008), whereas dopamine receptor blockade in the shell has minimal effects on similar behaviors (Nicola, 2010). This indicates that NAc core and shell have distinct roles in task performance. Indeed, lesions of the core but not shell reduce performance in different incentive-cue responding tasks (Di Ciano and Everitt,

\footnotetext{
Received Dec. 10, 2010; revised March 17, 2011; accepted March 21, 2011

Author contributions: F.A., A.G., S.M.N., and H.L.F. designed research; F.A. and A.G. performed research; F.A. and A.G. contributed unpublished reagents/analytic tools; F.A. and A.G. analyzed data; F.A., A.G., S.M.N., and H.L.F. wrote the paper.

This work was supported by National Institutes of Health Grant DA019473 to S.M.N. and by funds provided by the state of California for medical research on alcohol and substance abuse through the University of California, San Francisco. The authors are grateful to Dr. V. Kharazia for histology work.

*F.A. and A.G. contributed equally to this work.

Correspondence should be addressed to Frederic Ambroggi, Ernest Gallo Clinic and Research Center, University of California, San Francisco, 5858 Horton Street, Suite 200, Emeryville, CA 94608. E-mail: fambroggi@gallo.ucsf.edu. DOI:10.1523/JNEUROSCI.6491-10.2011

Copyright $\odot 2011$ the authors $\quad 0270-6474 / 11 / 316820-11 \$ 15.00 / 0$
}

2001; Di Ciano et al., 2001, 2008; Ito et al., 2004; Floresco et al., 2008; Chaudhri et al., 2010). We therefore investigated the differential contribution of NAc core and shell to behaviors in the DS task.

A subpopulation of NAc neurons responds to reward-predictive cues. The magnitude of these responses correlates with both the predictive value of the cue and the action elicited by the cue (Nicola et al., 2004b; Taha et al., 2007; Ito and Doya, 2009; Roesch et al., 2009). Furthermore, inactivating structures that provide afferent input to the NAc, such as the ventral tegmental area (VTA), the basolateral amygdala (BLA), and the dorsal prefrontal cortex (PFC), disrupts both responses in NAc neurons and behaviors elicited by reward-predictive cues (Yun et al., 2004b; Ambroggi et al., 2008; Ishikawa et al., 2008a,b). These observations strongly support the hypothesis that incentive-cue-elicited firing responses in NAc neurons promote reward-seeking behavior. However, they leave open the question of which neuronal firing patterns normally inhibit reward-seeking actions during intervals when reward is not available.

The differential contribution of the NAc core and the shell to reward-seeking behavior raises the possibility that their constituent neurons encode different information. Consistent with this idea, NAc core and shell receive different afferent projections (Groenewegen et al., 1999). On the other hand, some brain regions project to both the NAc core and shell (Voorn et al., 2004; Fields et al., 2007; Humphries and Prescott, 2010). Consequently, NAc core and shell neurons could encode similar information but have different effects on behavior depending on the downstream regions they innervate. Indeed, NAc core and shell project 
to distinct and almost non-overlapping downstream structures (Groenewegen et al., 1999; Voorn et al., 2004; Nicola, 2007; Humphries and Prescott, 2010; Tripathi et al., 2010). The difference of outputs raises the possibility that similar information encoded in the NAc core and the shell could play markedly different roles in behavior. To address this issue, we recorded the activity of NAc core and shell neurons during the DS task.

\section{Materials and Methods}

\section{Animals}

The subjects were male Long-Evans rats (Harlan Sprague Dawley) weighing $\sim 350 \mathrm{~g}$ on arrival and individually housed on a $12 \mathrm{~h}$ light/dark cycle. Experiments were conducted during the dark phase. After receipt, rats were allowed at least 1 week of ad libitum food and water, followed by 1 week of restricted food before training. Throughout all experiments, food restriction was adjusted daily at the end of experimental manipulations to maintain the rats at $\sim 90 \%$ of their initial body weight. Animal handling and experiments conformed to National Institutes of Health and Ernest Gallo Clinic and Research Center animal care and use policies.

\section{DS task}

The behavioral pharmacology study was conducted in standard operant chambers $(23.5 \times 30.5 \mathrm{~cm})$ and the electrophysiological study was conducted in larger cages $(40.6 \times 40.6 \mathrm{~cm})$. All cages contained two retractable levers located on one wall of the chamber (one on each side of a reward receptacle), two house lights, a white noise speaker, and a tone speaker (Med Associates). Liquid sucrose reward was delivered into a well in the reward receptacle by a syringe pump. The DS task structure was similar to our previous experiments (Nicola et al., 2004b; Yun et al., 2004a,b). Rats were run daily on the DS task for 1 or $2 \mathrm{~h}$ (for behavioral and electrophysiological studies, respectively). Two tones, the DS and the nonrewarded stimulus (NS), were presented on a variable interval schedule with an average interval of $30 \mathrm{~s}$. Pressing one of the two levers (designated the active lever, randomly chosen across rats) during DS presentation resulted in the delivery of $50 \mu \mathrm{l}$ of $10 \%$ sucrose into the reward receptacle and termination of the DS tone. Each DS lasted for up to $10 \mathrm{~s}$, and each NS lasted $10 \mathrm{~s}$. Responding on either lever during the NS or in the absence of the DS was never rewarded. The cues were either an intermittent $4 \mathrm{kHz}$ tone ( $40 \mathrm{~ms}$ on and $50 \mathrm{~ms}$ off) or a siren tone (ramped from 4 to $8 \mathrm{kHz}$ with a $400 \mathrm{~ms}$ period). Tones were randomly assigned to be the DS or the NS across rats.

Over the course of training, animals' responding to the DS increased, while NS responding and uncued lever pressing decreased. Surgeries were performed when the rats reached criterion performance of $>80 \%$ DS response ratio and $<20 \%$ NS response ratio (defined as the proportion of all DSs or NSs in the session to which the animal responded).

\section{Surgeries}

For the behavioral study, rats were bilaterally implanted with microinjection guide cannulae (27 gauge, Plastics One) in the NAc shell ( $\mathrm{AP}+1.6, \mathrm{ML} \pm 0.8$, $\mathrm{DV}-6.0 \mathrm{~mm}$ relative to bregma) and NAc core (AP $+1.6, \mathrm{ML} \pm 2$, and $\mathrm{DV}$ $-6.0 \mathrm{~mm}$ ).

For electrophysiological recordings, 2 arrays of 8 electrodes (NB Labs, $50 \mu \mathrm{m}$ stainless steel wires arranged in 2 rows of 4 ) were attached to a microdrive device that allowed the entire arrays to be lowered by $80 \mu \mathrm{m}$ increments. Target coordinates of the medioposterior electrode of each array were as follows: core, $\mathrm{AP}+1.2, \mathrm{ML} \pm 2.0$, and $\mathrm{DV}-6$ to $-8.5 \mathrm{~mm}$; and shell, AP $+1.2, \mathrm{ML}, \pm 0.8$, and $\mathrm{DV}-6$ to $-8.5 \mathrm{~mm})$.

Animals were anesthetized with isoflurane (5\%) and placed in a stereotaxic apparatus. Anesthesia was maintained with isoflurane (0.5$2.0 \%$ ) during surgery. Microdrives were secured to the skull with bone screws and dental acrylic, and wire obturators were inserted into the guide cannulae; the ends of the obturators were flush with the ends of the guide cannulae. Rats were given at least $7 \mathrm{~d}$ of recovery before being retrained on the DS task and habituated to the handling procedures.

\section{Microinjections}

To block excitatory transmission in the NAc, we used a mixture of the AMPA and NMDA antagonists 6-cyano-7-nitroquinoxaline-2,3-dione
(CNQX) and 2-amino-5-phosphonopentanoic acid (AP5), which inhibit NAc medium spiny neuron activity because these neurons require glutamatergic input to fire (Horne et al., 1990; Pennartz et al., 1991; Nicola et al., 1996; Nicola et al., 2000). CNQX (4 mg/ml) and AP5 (8 mg/ml) were dissolved separately in $1 \mathrm{M} \mathrm{NaOH}$. After adjusting the $\mathrm{pH}$ to 7.0 with $1 \mathrm{M}$ $\mathrm{HCl}$, the solutions were combined.

To inject animals, the obturators were removed and 30 gauge injector cannulae were inserted into the guides. Injectors extended $1.5 \mathrm{~mm}$ below the tip of the cannula. A $0.5 \mu$ l volume of CSF or the mixture CNQX/AP5 ( 1 and $2 \mu \mathrm{g}$, respectively) was injected over $2 \mathrm{~min}$. After a $1 \mathrm{~min}$ postinjection period, the injectors were gently removed, the obturators were replaced, the animal was immediately placed into the behavioral chamber, and the behavioral session began. CSF and the mixture CNQX/AP5 were injected on different days (with at least one intervening day with no injection), in random order, in the same animals.

\section{Electrophysiology}

Electrophysiological recording was conducted as described previously (Nicola et al., 2004b; Ambroggi et al., 2008). Animals were connected to the recording apparatus (Plexon), which consisted of a headstage with operational amplifiers, a cable, and a commutator to allow the animal free movement within the chamber. Rats were run for $2 \mathrm{~h}$ daily sessions of the DS task. The microdrive carrying the electrode arrays was lowered by 80 or $160 \mu \mathrm{m}$ at the end of each session to get a new set of neurons every day.

Spike sorting. Isolation of individual units was performed offline with Offline Sorter (Plexon) using principal component analysis. Only units with well defined waveforms were included in this study. Interspike interval distribution, cross-correlograms, and autocorrelograms were used to ensure that single units were isolated. Additionally, only units in which waveform characteristics were constant over the entire recording session were included in this study.

Determination of the optimal bin size. The size of the bins greatly influences the information contained in peristimulus time histograms (PSTHs). On one hand, the bin size should be sufficiently small to provide the temporal resolution needed to detect brief changes in the firing. On the other hand, the degree of firing noise limits the smallest possible bin size that maintains a minimum signal-to-noise ratio. Larger bin sizes reduce the noise variance by averaging the firing over time. Therefore choosing the optimal bin size is a trade-off between temporal accuracy (gained with smaller bin sizes) and the amount of noise (reduced for larger bin sizes) in the firing rate measure. We developed a method to find the optimal bin size for each neuron by using the Akaike information criterion (AIC), defined as follows:

$$
A I C=T \log _{e}\left(\frac{S S E}{T}\right)+2 P
$$

where

$$
S S E=\sum_{t=1}^{T}(\hat{\lambda}(t, \Delta)-\hat{\lambda}(t, 1))^{2} .
$$

Here $\hat{\lambda}(t, 1)$ represents the firing rate estimate with the smallest bin size considered (here $10 \mathrm{~ms}$ ) and $\hat{\lambda}(t, \Delta)$ is the firing rate estimate with a larger bin size equal to $\Delta . P$ is an integer representing the number of bins used for the PSTH when the bin size is equal to $\Delta$ and $T$ is the maximum of number of bins used when the smallest bin size is chosen. The sum of the squared error (SSE) is minimized when $\Delta$ approaches 1 , in which case the number of parameters $P$ is equal to T. However, because AIC includes a term that penalizes large parameter numbers, AIC in most cases is minimized at intermediate bin sizes that result in lower numbers of parameters compared to $T$. This is not accidental, as very small bin sizes can result in overfitting the data and are not necessarily optimal. This can be verified by large AIC costs associated with very small bin sizes (usually $<40 \mathrm{~ms}$ ). Because the optimal bin size is usually rather large for most neurons $(\sim 0.1-1 \mathrm{~s})$, and because the AIC commonly shows a fast reduction over small bin sizes followed by slow changes around the optimal bin size, we also made PSTHs using the smallest possible bin size that showed less than a $10 \%$ change from the optimal AIC value. This bin size is 
referred to as the deflection point bin size and was on average $60 \mathrm{~ms}$ across our neuronal population.

Response detection. PSTHs constructed around the behavioral events, with the optimal bin size, were used to detect excitations and inhibitions and the time at which they occurred. The $10 \mathrm{~s}$ period before cue onset was used as a baseline period. Excitation and inhibition to each event was determined by the presence of at least one bin above (for excitations) or below (for inhibitions) the $99 \%$ confidence interval of the baseline during the analysis window for each event. Each unit was assigned, based on its firing pattern, to at least one of the subsets of neurons exhibiting the response types. Onset detection was performed using a telescopic approach. We selected the first significant bin using the optimal size and searched within this time window for the first bin using the deflection point bin size that was beyond $99 \%$ confidence interval of the baseline made with this new bin size. If found, we then repeated the search within this deflection point bin, for the first $10 \mathrm{~ms}$ bin that was beyond $99 \%$ confidence of the baseline made with $10 \mathrm{~ms}$ bin size. With this method, the onset is defined by the highest valid resolution for each PSTH. Response duration was computed by finding the first bin after the detected onset that fell into the $99 \%$ confidence interval of the baseline based on the optimal bin size only. The choice of different strategies used for onset and duration detection was based on our observation that the telescopic approach and the optimal bin size tend to be more accurate for onset and duration estimation, respectively. For some neurons, the response types, onsets, and durations were also visually inspected afterward to ensure accuracy.

Deconvolution. We have shown previously that PSTHs made for temporally close events do not allow one to accurately account for the neuronal responses to each individual event. We have developed and validated an iterative deconvolution methodology that can parse out the neuronal responses of each individual event from the PSTHs (Ghazizadeh et al., 2010). During DS trials, four events usually happen within a short period of time, namely, DS, lever press, receptacle entry, and receptacle exit. PSTHs made for each of the events can potentially be distorted due to the presence of the other events that are correlated with it in time. This can specifically pose a problem for comparing the DS to the NS firing, which is not normally followed by other events. For each neuron, we deconvolved single-event responses by using the maximum number of iterations that had a cross-validation error lower than the PSTHs (average iteration of 4.95 for the entire dataset) (Ghazizadeh et al., 2010). Therefore, instead of the PSTHs, we used, when appropriate, deconvolved responses to report the average activity. The area under the curve (AUC, integrated from response onset to offset) and the peak were obtained from the deconvolved responses and used for statistical comparisons.

Data transformation and plotting. Color-coded maps were constructed with optimal bins and average PSTHs across neurons were constructed with the deflection point bins. Before averaging, the firing rate of each neuron during each bin was transformed to a $Z$-score: $\left(F_{i}-F_{\text {mean }} / F_{\text {sd }}\right.$ where $F_{i}$ is the firing rate of the $i$ th bin of the PSTH, and $F_{\text {mean }}$ and $F_{\text {sd }}$ are, respectively, the mean and the SD of the firing rate during the $10 \mathrm{~s}$ preceding cue onset.

\section{Histology}

Animals were deeply anesthetized with pentobarbital and perfused intracardially with saline and $4 \%$ formalin (plus 3\% ferrocyanide for rats with electrode arrays). Brains were removed, sectioned $(40 \mu \mathrm{m})$, and stained for Nissl substance to locate injection or recording sites (labeled by passing a DC current through each electrode before perfusion).

\section{Statistical analysis}

$t$ tests or ANOVAs followed by Newman-Keuls post hoc tests were used when appropriate. A permutation test was used to compare proportions. All results were considered significant at $p<0.05$.

\section{Results}

All rats in this study were trained on the DS task (Fig. $1 A$ ). One of two auditory cues, the rewarded DS and the NS, was randomly presented on average every $30 \mathrm{~s}$. During a DS presentation, rats

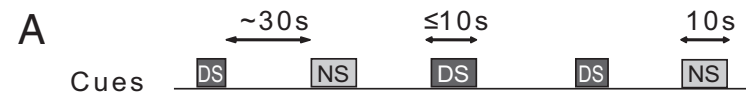

$\begin{array}{lll}\text { Active LPs } 1 & 1 & 1\end{array}$

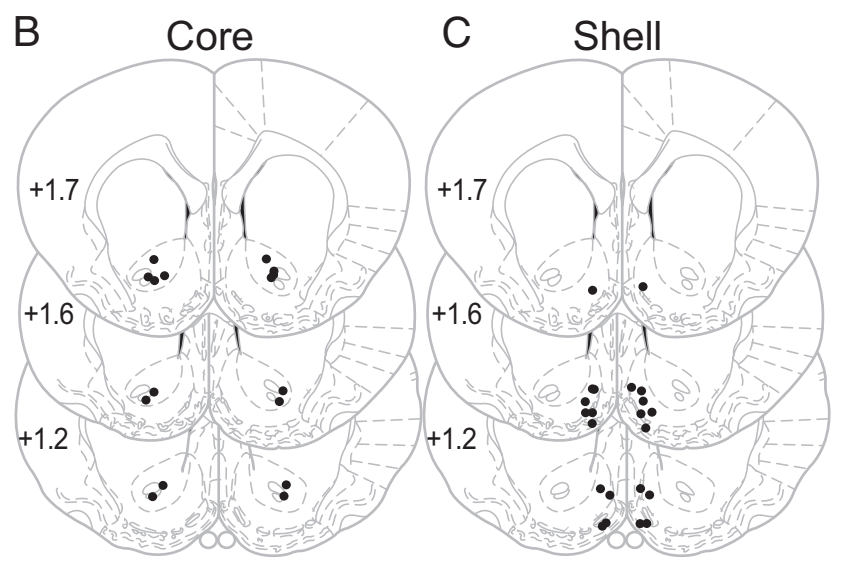

Figure 1. Schematic of the DS task and histology. A, Two cue tones [up to $10 \mathrm{~s}$ for the rewarded discriminative stimulus (DS); 10 s for the unrewarded stimulus (NS)] were randomly presented on a variable-interval schedule with an average interval of $30 \mathrm{~s}$. A lever press was required during DS presentation to terminate the DS and cause the delivery of a $10 \%$ sucrose reward into an adjacent receptacle. $\boldsymbol{B}, \boldsymbol{C}$, Histological reconstruction of injector placements in the core $(\boldsymbol{B})$ and the shell $(\boldsymbol{C})$.

pressed the active lever, which turned off the auditory cue and delivered the reward. They then quickly entered the reward receptacle to retrieve the reward. After consumption of the sucrose reward, rats would exit the reward receptacle to await the next cue presentation. During the intertrial interval and following the NS, rats also intermittently pressed the active and inactive levers or entered the reward receptacle, despite the fact that those actions were not rewarded.

\section{Pharmacological inactivation of the NAc core and shell}

Two groups of animals with guide cannulae in either the NAc core or shell (Fig. 1B,C) received microinjections of CSF and a mixture of glutamate antagonists CNQX/AP5 (1 and $2 \mu \mathrm{g}$, respectively, in $0.5 \mu \mathrm{l}$ ) on separate days.

\section{Incentive-cue responding}

The DS response ratio (defined as the proportion of DSs the animals responded to), the number of rewarded active lever presses and the latency to lever press after DS presentation are related to the strength of the incentive cue (the DS) to promote behavior to obtain the reward. All measures were significantly affected by the injections (Fig. 2A-C) (two-way ANOVAs, structure $F_{(1,17)}>6.7, p<0.02$, injection $F_{(1,17)}>39, p<0.001$, structure $\times$ injection $F_{(1,17)}>22, p<0.001$ for all variables). Inactivation of the NAc core but not the shell significantly reduced the DS ratio (Fig. $2 A$ ) (Newman-Keuls, $p<0.001$ ) and increased the latency to lever press after DS (Fig. $2 B$ ) (NewmanKeuls, $p<0.001)$.

\section{Disinhibition of active lever presses}

The above results demonstrate that the NAc core but not shell is required for incentive-cue responding. In contrast, both NAc core and shell inactivation produced significant behavioral disinhibition. The NS ratio was increased following inactivation but did not depend on the structure (Fig. 2A) (two-way ANOVA, structure $F_{(1,17)}=2.2, p=0.15$, injection $F_{(1,17)}=7.4, p=0.01$, 

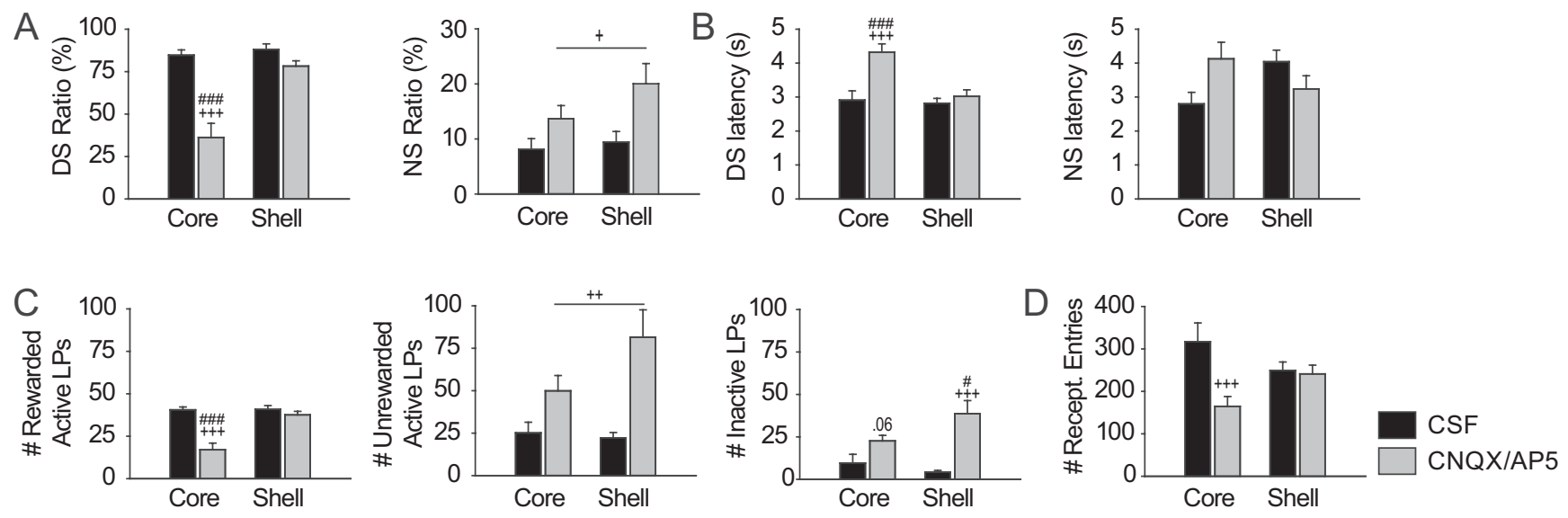

Figure 2. Effect of pharmacological inactivation (CNQX/AP5, 1 and $2 \mu \mathrm{g} / 0.5 \mu \mathrm{l}$, respectively) of the NAc core and shell on the DS task. $A$, Average DS (left) and NS (right) response ratios (proportion of DSs or NSs during which the animal pressed the lever). B, Average DS (left) and NS (right) response latencies (time from cue onset to the lever press). $C$, Average number of rewarded (i.e., occurring during DS presentations, left) and unrewarded (outside DS presentations, middle) lever presses on the active and total number of lever presses on the inactive lever (right). $\boldsymbol{D}$, Average number of reward receptacle entries. ${ }^{+} p<0.05,{ }^{++} p<0.01,{ }^{+++} p<0.001$ compared to CSF injections and ${ }^{\#} p<0.05,{ }^{\# \# \#} p<0.001$ compared to shell injections.

structure $\times$ injection $\left.F_{(1,17)}=0.7, p=0.4\right)$. Similarly, the number of unrewarded (active) lever presses (those occurring outside the DS presentations) was also increased after inactivation of either structure (Fig. 2C) (two-way ANOVA structure $F_{(1,17)}=$ $1.7, p=0.2$, injection $F_{(1,17)}=15.2, p=0.002$, structure $\times$ injection $F_{(1,17)}=2.6, p=0.1$.

The fact that unrewarded active lever presses were increased after NAc inactivation raises the question of the temporal specificity of the increase during the NS. Do rats press the active lever in response to the NS or is the NS ratio increase primarily due to a nonspecific general increase in lever pressing? To address this question, we analyzed the frequency of lever pressing during different time windows (Fig. $3 A$ ): during the intertrial interval (spontaneous), during the DS, during the NS, and in the $10 \mathrm{~s}$ after the rats consumed the reward and exited the receptacle (postreward). The injections affected the frequency of active lever presses differently (Fig. $3 B$ ) (three-way ANOVA, structure $F_{(1,68)}=12.8, p=0.001$, injection $F_{(1,68)}=3.7$, $p=0.06$, window $F_{(3,68)}=211, p<0.001$, structure $\times$ injection $\times$ window $\left.F_{(3,68)}=7.2, p<0.001\right)$. NAc core inactivation reduced lever pressing during the DS but did not significantly increase lever pressing in the spontaneous or NS windows. Compared to CSF injection, inactivation of the core increased lever pressing immediately after collection of the reward (postreward window) to a greater degree than in the spontaneous window. NAc shell inactivation had greater disinhibitory effects: active lever pressing was increased during the spontaneous, NS, and postreward windows. Furthermore, the increase in active lever pressing in these two latter time windows was stronger than that during the spontaneous window. This shows that the increase in NS ratio caused by shell inactivation is the combination of a general increase in lever pressing and the removal of a temporally specific NS-driven inhibition of responding.

Because the disinhibitory effect of shell inactivation consisted, in part, of a specific increase in responding during the NS coupled with no effect on DS responding, one possibility is that shell inactivation reduces animals' ability to discriminate between the two cues. However, animals clearly continued to discriminate DS from NS after shell inactivation, as they responded to $80 \%$ of DSs and only $20 \%$ of NSs (Fig. $2 A$ ). Furthermore, shell inactivation increases responding in the absence of cues (Figs. 2C, 3B). These results are more consistent with a contribution of the NAc shell to response inhibition than to cue discrimination.
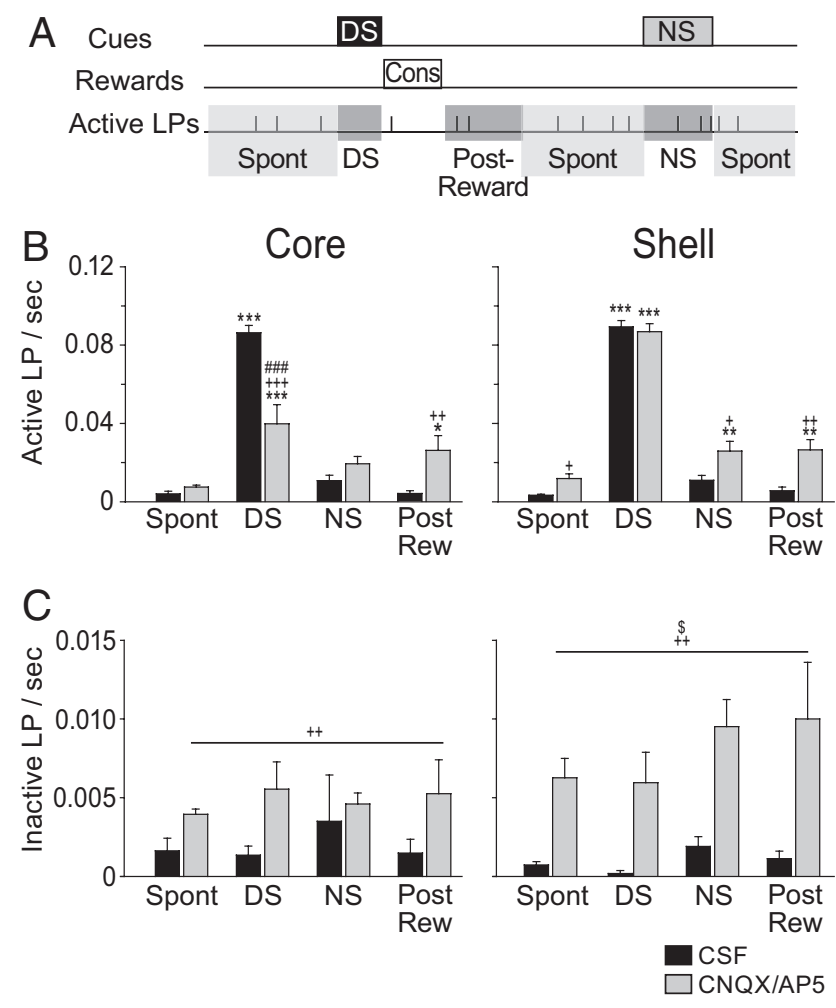

Figure 3. Time window analysis of lever pressing after pharmacological inactivation of the NAc core and shell. $A$, Schematic illustrating the time windows in which the frequency of lever pressing was analyzed. DS and NS windows correspond to the entire duration of the cues. The postreward window corresponds to the 10 s following the consumption of the reward. The spontaneous window (spon) corresponds to the remaining time. $\boldsymbol{B}, \boldsymbol{C}$, Frequency of active $(\boldsymbol{B})$ and inactive $(\boldsymbol{C})$ lever pressing in the different time windows after CSF or CNQX/AP5 injections in the core (left) or the shell (right). ${ }^{*} p<0.05,{ }^{* *} p<0.01$, ${ }^{* * *} p<0.001$ compared to the spontaneous window; ${ }^{+} p<$ $0.05,{ }^{++} p<0.01,{ }^{+++} p<0.001$ compared to CSF injections and ${ }^{\#} p<0.05,{ }^{\# \#} p<0.01$, ${ }^{\# \# \# p}<0.001$ compared to shell injections, ${ }^{\$} p<0.05$ structure $\times$ injection effect.

\section{Disinhibition of inactive lever presses}

Inactivation of the NAc also increased the number of presses on the inactive lever (Fig. 2C) (two-way ANOVA, structure $F_{(1,17)}=$ $0.8, p=0.4$, injection $F_{(1,17)}=25, p<0.001$, structure $\times$ injection $\left.F_{(1,17)}=5.12, p=0.03\right)$. NAc shell inactivation significantly increased inactive lever presses (Newman-Keuls $p<0.001$ ), 
Table 1. Total number of neurons recorded per rat in the core and the shell

\begin{tabular}{clrr}
\hline Rat & Target & Core & Shell \\
\hline 1 & Core and shell & 57 & 39 \\
2 & Core and shell & 24 & 34 \\
3 & Core and shell & 144 & 34 \\
4 & Core and shell & 58 & 10 \\
5 & Core and shell & 14 & 15 \\
6 & Core and shell & 28 & 63 \\
7 & Core & 73 & 0 \\
8 & Core & 8 & 0 \\
9 & Core & 72 & 0 \\
10 & Shell & 0 & 198 \\
11 & Shell & 0 & 59 \\
12 & Shell & 0 & 53 \\
Total & & 478 & 505 \\
Total $>0.03 \mathrm{~Hz}$ & & 456 & 484 \\
\hline
\end{tabular}

whereas NAc core inactivation produced a nonsignificant trend toward an increase (Newman-Keuls, $p=0.06$ ).

When analyzed in different time windows, the number of presses on the inactive lever after NAc inactivation showed an injection effect and a structure $\times$ injection effect (Fig. 3C) (three-way ANOVA, structure $F_{(1,68)}=1.5, p=0.2$, injection $F_{(1,68)}=35, p<0.001$, window $F_{(3,68)}=1.1, p=0.4$, structure $\times$ injection $\times$ window $\left.F_{(3,68)}=0.4, p=0.7\right)$. The increase in inactive lever pressing is stronger for shell than core inactivation (Newman-Keuls on structure $\times$ injection interaction effect, $p=$ $0.01)$. However, in contrast to active lever presses, we found no time window effect. This indicates that the disinhibition of inactive lever presses is independent of discrete cues or reward receipt; i.e., that there is no temporally specific relation of NAc-mediated inhibition of inactive lever pressing to any specific component of the task.

\section{Reward receptacle entries}

The total number of receptacle entries was affected by inactivations (Fig. 2D) (two-way ANOVA, structure $F_{(1,17)}<0.1$, $p=0.9$, injection $F_{(1,17)}=13, p=0.002$, structure $\times$ injection $\left.F_{(1,17)}=10.5, p<0.005\right)$ with a reduction after NAc core (Newman-Keuls, $p<0.001$ ) but not shell inactivation (Newman-Keuls, $p>0.05)$. Because the number of receptacle entries is more than five times larger than the number of rewards earned (compare Fig. 2C,D), the prominent reduction of entries after NAc core inactivation cannot be due solely to reduced DS responding, but also reflects lower motivation to check the reward receptacle in the absence of cues.

\section{Electrophysiological activity of NAc core and shell neurons}

We recorded 983 neurons located in the NAc of 12 rats (Table 1) during the DS task in $>166$ sessions. After exclusion of neurons with a very low baseline firing rate $(<0.03 \mathrm{~Hz}), 456$ and 484 neurons located, respectively, in the NAc core and shell remained in our sample for further analysis (Fig. 4). A small proportion of the neurons $(n=44)$ (Fig. 4 , dark gray dots) was located in a boundary area where the NAc shell and the ventral pallidum were indistinguishable either anatomically or electrophysiologically. Excluding neurons located in this area had no impact on the significance of the effects reported below (data not shown). Therefore, since many of these neurons were probably shell neurons, we performed all our analyses on the entire shell dataset.

Behaviorally, rats responded more and faster to the DS than to the NS (DS ratio $84 \pm 0.7 \%$, NS ratio $5.1 \pm 0.4 \%, p \ll 0.001$; DS latency $2.9 \pm 0.05 \mathrm{~s}$, NS latency $4.1 \pm 0.16 \mathrm{~s}, p \ll 0.001)$. The

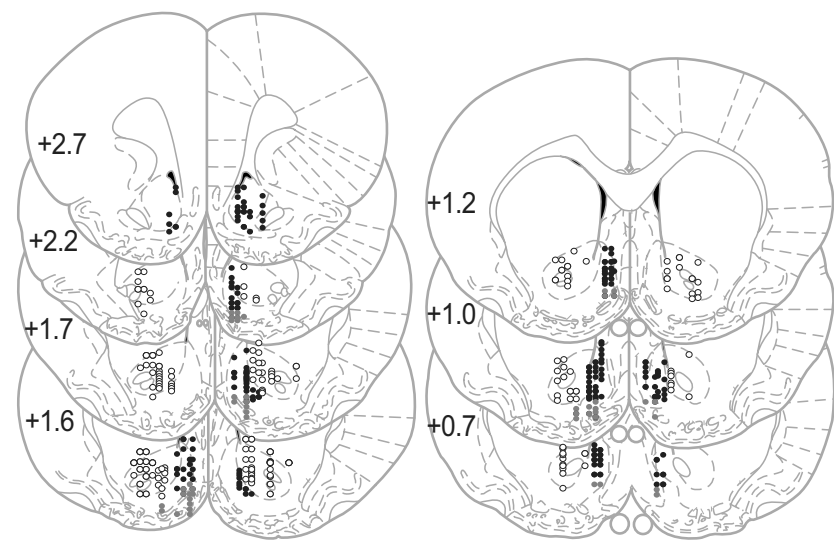

Figure 4. Histological reconstruction of electrode placements in the NAc core (open circles) and shell (black and gray dots). Gray dots represent the electrode sites close to the ventral pallidum that were included in the shell data.

basal firing rate in the NAc core and shell was similar $(3.56 \pm 0.24$ and $3.65 \pm 0.34 \mathrm{~Hz}$, respectively, $\left.t_{(938)}=0.21, p=0.83\right)$. As previously shown in the DS task, different but overlapping subpopulations of NAc neurons respond to cues, operant actions, reward consumption, and exit of the reward receptacle (Nicola et al., 2004b). Here, we examine the distributions of each of these firing responses across NAc core and shell neurons.

\section{Cue-related responses in NAc neurons}

We found cue-related responses in both the core and the shell. However, DS excitations were more frequent in NAc core than shell (permutation test, $p<0.007$ ) (Fig. 5A). This difference was not seen for NS excitations (permutation test, $p>0.3$ ). Neurons with cue-evoked inhibitions were equally common in the NAc core and shell (permutation tests, $p>0.26$ ). For both DS and NS neuronal responses, onset latencies depended on the type of response (excitation/inhibition) and the structure (Fig. 5B) (twoway ANOVA, for DS structure $F_{(1,286)}=0.35, p=0.55$, response type $F_{(1,286)}=21.7, p<0.001$, structure $\times$ response type $F_{(1,286)}=4.65, p=0.03$; for NS, structure $F_{(1,56)}=4.82$, $p=0.03$, response type $F_{(1,56)}=16.56, p=0.002$, structure $\times$ response type $\left.F_{(1,56)}=5.28, p=0.03\right)$. The onset latencies for both DS and NS excitations did not differ between core and shell (Newman-Keuls $p>0.27$ ). Cue-evoked inhibitions however, had longer latencies in shell than core (for DS and NS, NewmanKeuls $p<0.05)$. Analysis of the duration of DS responses revealed a main effect of response type $\left(F_{(1,286)}=14.5, p<0.005\right.$ and a structure $\times$ response type interaction $\left(F_{(1,286)}=9.2, p<0.005\right)$. Notably, DS inhibitions in the NAc core were significantly longer than all the other response types (Newman-Keuls $p<0.005$ ). Indeed, some NAc core neurons were inhibited for $>10 \mathrm{~s}$ (Fig. $5 C$ ). In contrast, in the shell, the duration of inhibitions and excitations was similar (Newman-Keuls $p=0.58$ ). Importantly, rats pressed the lever an average of $2.9 \pm 0.05 \mathrm{~s}$ after the DS was presented (Fig. 4A). This implies that many inhibitions in the core encompassed the lever press and reward consumption. This effect contrasts with the durations of other DS response types that usually terminated before or around the time of lever press. We then sorted the neurons according to whether they responded to one of the cues or both (Fig. 6A,B). Interestingly, despite similar proportions of NS excited neurons, the overlap between DS and NS excitations was larger in the NAc core than in the shell (permutation test, $p<0.05$ ).

The fact that some neuronal responses span multiple task events is a potential confound for analyzing event-related neu- 
A

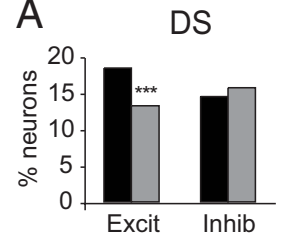

$\mathrm{B}$
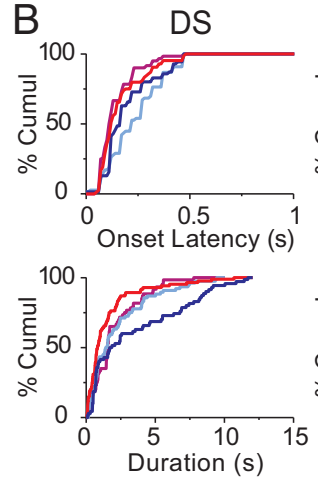

NS
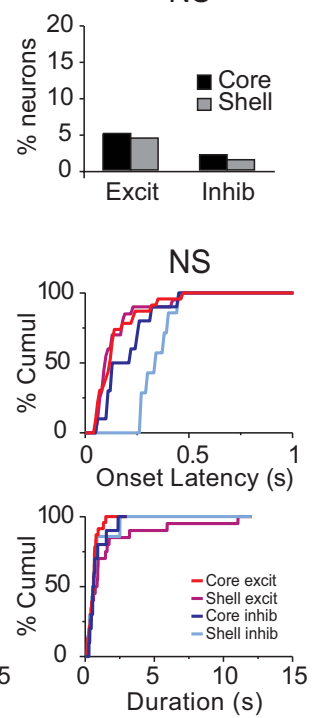

C
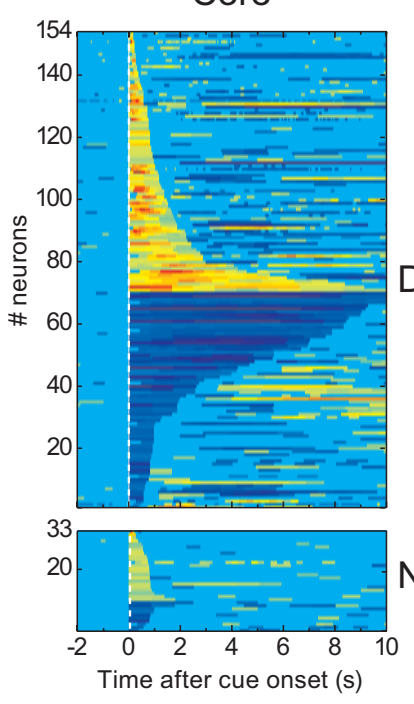

Shell

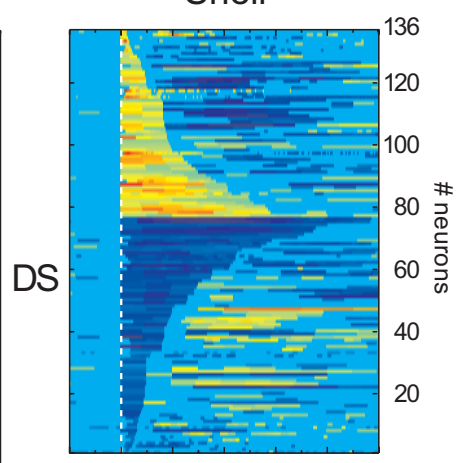

Z-score

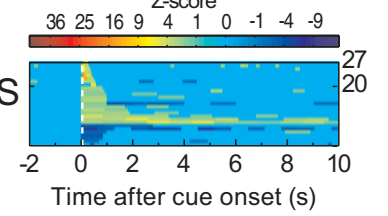

Figure 5. Neuronal responses to the DS and NS in the NAc. $A$, Percentage of excitation and inhibition in response to the DS (left) and NS (right). $\boldsymbol{B}$, Cumulative percentage of onset response latency (top) and response duration (bottom) for DS (left) and NS (right). C, Normalized (as Z-score) and color-coded PSTH showing all DS- (top) and NS- (bottom) responsive neurons. Each line represents the PSTH of a single neuron. Cue-excited neurons are displayed at the top and cue-inhibited neurons at the bottom. Within each category, neurons are sorted by response durations (shorter responses at the top for excitations and at the bottom for inhibitions). Deconvolution of PSTHs was not used for the analyses in this figure.

A Core
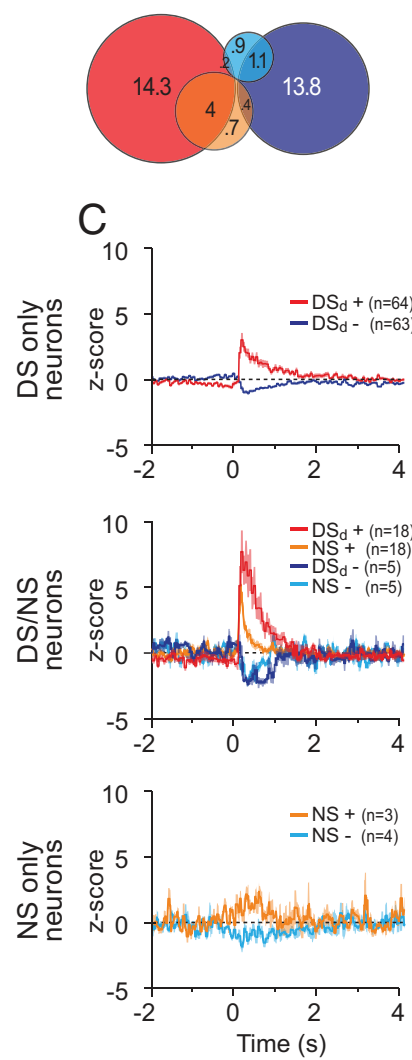

B

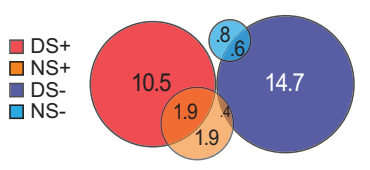

$\mathrm{D}$

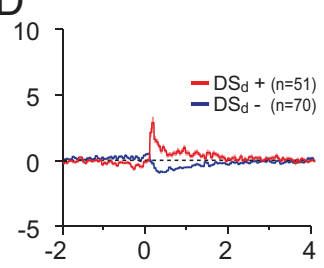

$\mathrm{E}$
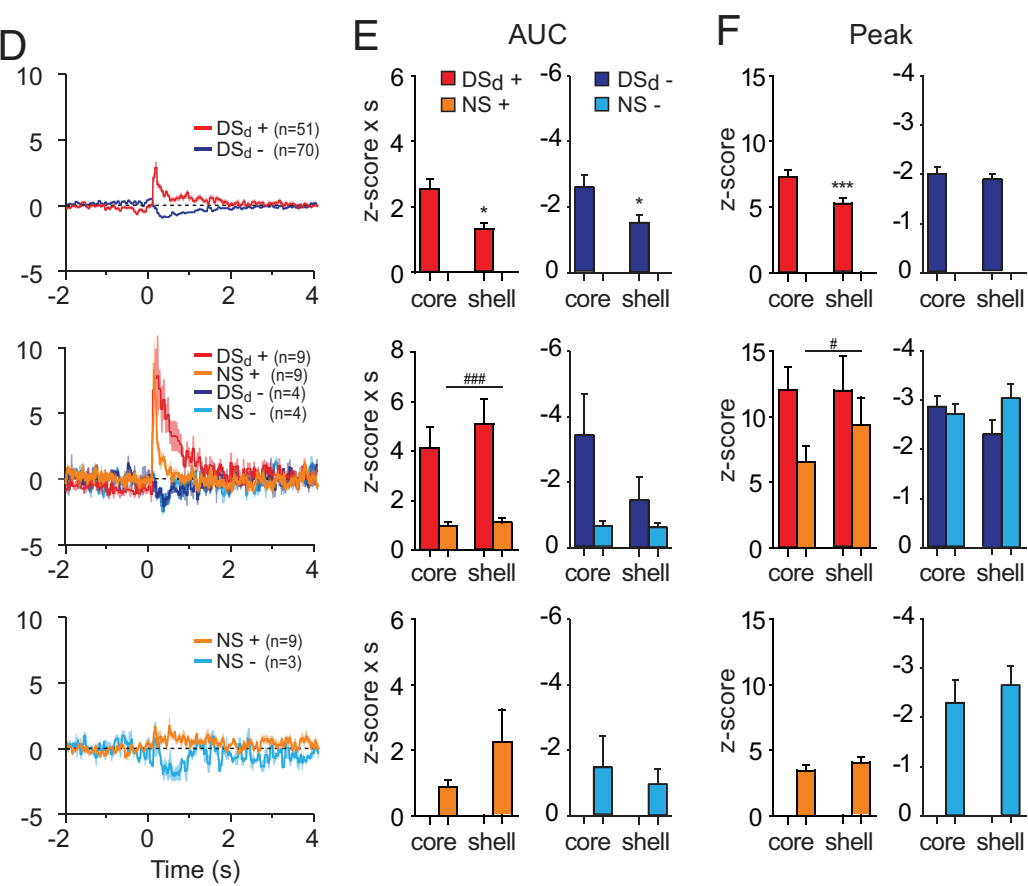

Figure 6. Overlaps between DS and NS responses. $A, B$, Venn diagram showing the proportion of DS-excited (red), DS-inhibited (dark blue), NS-excited (orange), and NS-inhibited (light blue) responses for the core $(\boldsymbol{A})$ and the shell $(\boldsymbol{B})$. The overlaps represent the neurons having two types of responses. $\boldsymbol{C}, \boldsymbol{D}$, Average responses to DS and/or NS in the core $(\boldsymbol{C})$ and the shell $(\boldsymbol{D})$. Top, Neurons responding only to DS. Middle, Neurons responding to both DS and NS with the same response direction (excitation or inhibition). Bottom, Neurons responding only to NS. $\boldsymbol{E}, \boldsymbol{F}$, AUC $(\boldsymbol{E})$ and peak $(\boldsymbol{F})$ of DS and/or NS responses. ${ }^{*} p<0.05$ structure effect, ${ }^{\# \#} p<0.001$ cue effect. 
ral responses. This is particularly true for responses to cues followed at short latency by actions. For example, most DSs were rapidly followed by a lever press, while NSs typically were not. A different firing pattern following either DS or NS could therefore be primarily related to lever press. We recently developed an analysis method for parsing the firing related to multiple sequential events. The procedure uses the natural variability in time between events to separate the firing that could be related to either or both events and thereby isolate the firing relative to a single event (Ghazizadeh et al., 2010). We applied this method to three events: DS, lever press, and reward receptacle exit. Because reward receptacle entry occurred at relatively constant latencies after lever presses (Nicola et al., 2004a; Nicola, 2010), we did not include reward receptacle entry in our analysis. The lever press related responses studied below could therefore be also related to receptacle entry.

Analysis of the neuronal population responsive only to the DS (Fig. $6 C-F$, top row) revealed that excitations and inhibitions were larger in the NAc core than in the shell (Fig. 6E, $F$, top row) (excitations $t_{(114)}<-2.0, p<0.05$ for both AUC and peak; inhibition $t_{(132)}=-2.4, p<0.05$ for AUC and $t_{(132)}=-0.7, p=$ 0.47 for peak). For the population of neurons excited by both cues, deconvolved DS (DSd) responses were considerably larger than NS responses in both the core and the shell (Fig. 6C-E, middle row) (two-way ANOVA, cue $F_{(1,25)}>4.2, p<0.05$, structure $F_{(1,25)}>0.52, p>0.47$, cue $\times$ structure interaction $F_{(1,25)}<$ $0.51, p>0.48$ for both AUC and peak). We found no significant effect for inhibitions (two-way ANOVA, $F_{(1,6)}<4, p>0.09$ ). The subpopulation of neurons responsive only to NS had small responses similar in core and shell (Fig. 6C-F, bottom row) (excitations $t_{(10)}<-0.64, p>0.47$ for both AUC and peak; inhibition $t_{(6)}<0.61, p>0.56$ for both AUC and peak) with less defined peaks. Excitation peaks appeared at considerably longer latencies than the other cue-related responses (excitations: 550 and $460 \mathrm{~ms}$ for NS responses of NAc core and shell, respectively, compared to $120 \mathrm{~ms}$ for all other cue responses in DS-only and DS/NS responsive neurons, $p>0.05$ ).

Overall, these results show that the DS is more robustly represented in NAc core than shell neuronal populations. Indeed, the proportion of DS excitations and the magnitude of both DS excitations and DS inhibitions are larger in the NAc core for neurons responding only to the DS. Notably, this is not the case for neurons coexcited by DS and NS; however, these neurons constitute a considerably smaller population than those responsive to DS only. Although the proportion of NS responding neurons is similar in core and shell, more shell neurons respond selectively to the NS.

NAc neuronal correlates of rewarded lever-press responses We then analyzed phasic responses occurring around rewarded active lever presses (Fig. 7, see Materials and Methods). Neurons inhibited during the lever press were more frequently found in the NAc shell than the core (Fig. 7A) (permutation test, $p=$ 0.027). Onset latencies of excitations and inhibitions were similar across structures (Fig. 7B) (two-way ANOVA structure $F_{(1,188)}<$ $0.1, p=0.8$, response type $F_{(1,188)}=0.1, p=0.7$, structure $\times$ response type $\left.F_{(1,188)}<0.1, p=0.8\right)$. Excitations were stronger in the NAc core than in the shell (Fig. $7 C-H)\left(\right.$ AUC, $t_{(74)}=4.47, p<$ 0.001 and peak $\left.t_{(74)}=3.31, p<0.005\right)$, but inhibitions were similar (AUC, $t_{(114)}=0.69, p=0.49$ and peak $t_{(114)}=0.94, p=$ 0.94). In conclusion, there is a reciprocal pattern of dominant neuronal representation of rewarded lever presses in the NAc
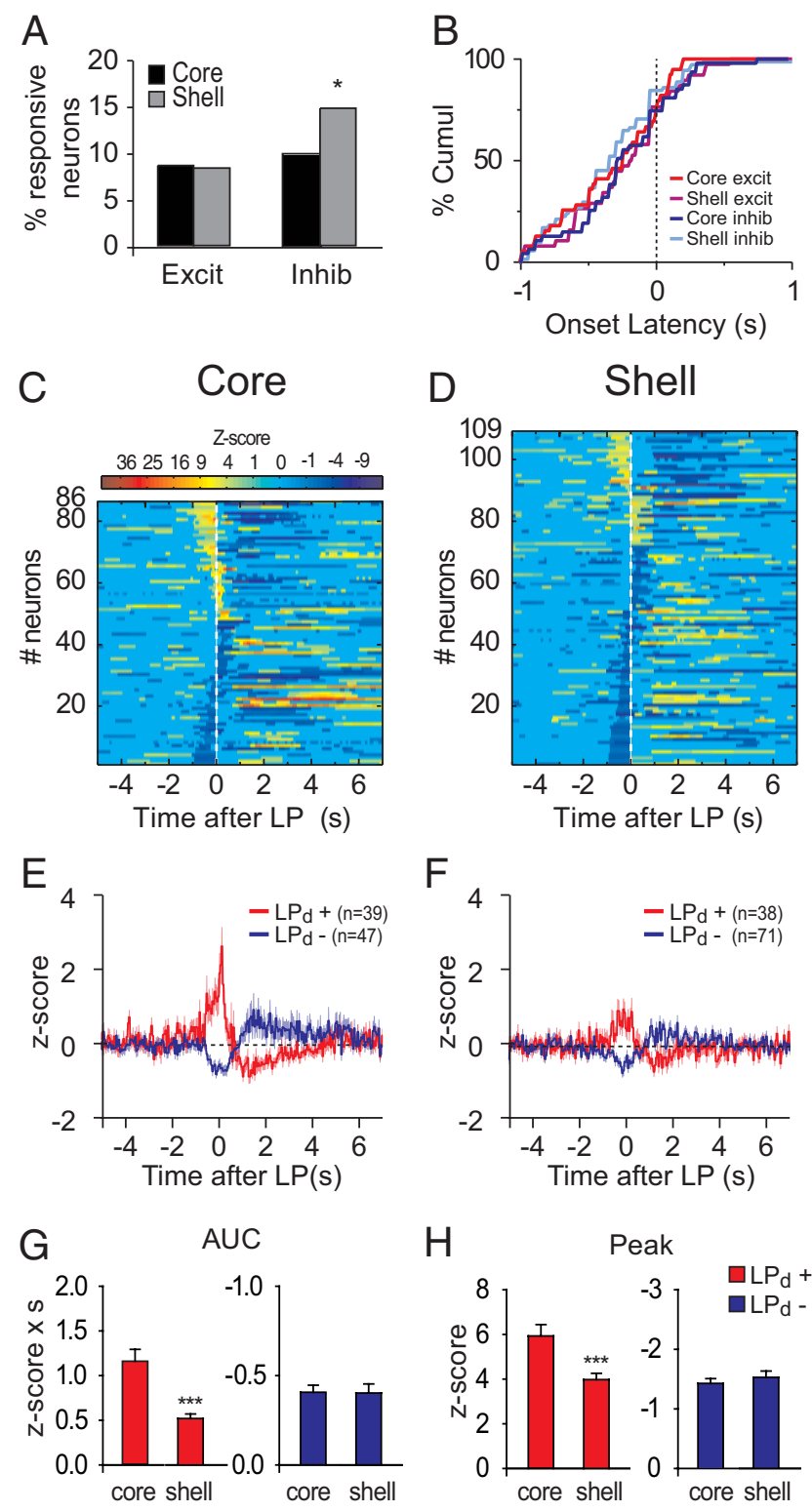

Figure 7. Neuronal responses to rewarded lever presses. $A$, Percentage of excitation and inhibition in response to the lever press in the core and the shell. $\boldsymbol{B}$, Cumulative percentage of onset latency of lever press-related responses. $\boldsymbol{C}, \boldsymbol{D}$, Normalized and color-coded PSTHs aligned to the lever press for core $(\boldsymbol{C})$ and shell $(\boldsymbol{D})$. Each line represents the PSTH of a single neuron. LP-excited neurons are displayed at the top and LP-inhibited neurons at the bottom. Within each category, neurons are sorted by response durations (shorter responses at the top for excitations and at the bottom for inhibitions). $\boldsymbol{E}, \boldsymbol{F}$, Average lever-press responses for core $(\boldsymbol{E})$ and shell $(\boldsymbol{F})$ neurons. $\boldsymbol{G}, \boldsymbol{H}$, Peak $(\boldsymbol{G})$ and AUC $(\boldsymbol{H})$ of lever-press responses. ${ }^{* *} p<0.01$ compared to core.

core and shell: stronger magnitude of excitations in the core but more frequent inhibitions in the shell.

\section{NAc responses to reward consumption}

Previous studies show that many NAc neurons respond during reward consumption (Nicola et al., 2004a; Roitman et al., 2005; Taha and Fields, 2005; Krause et al., 2010), although whether their firing changes encode reward valence or promote responding for reward has been debated (Roitman et al., 2005; Taha and Fields, 2005; Krause et al., 2010). Both NAc core and shell neurons were more often inhibited than excited during reward consumption (permutation test, $p<0.01$ for both core and shell) 
A

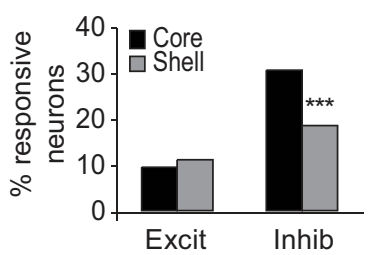

C

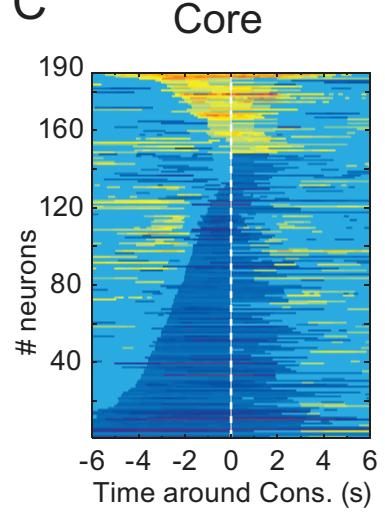

E

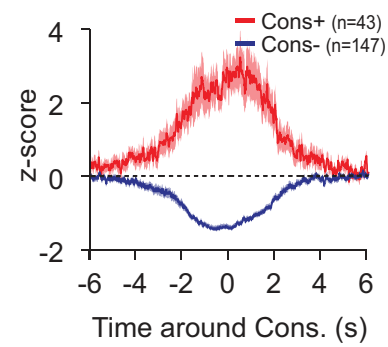

G AUC

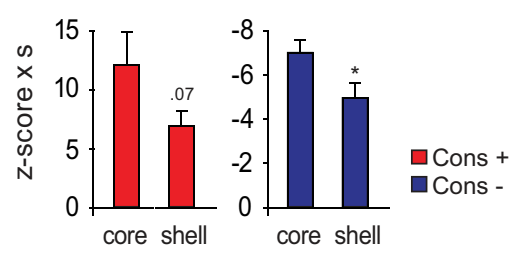

Figure 8. Neuronal responses to reward consumption. $\boldsymbol{A}$, Percentage of excitation and inhibition in response to the reward consumption in the core and the shell. $\boldsymbol{B}$, Cumulative percentage of response duration of consumption-related responses. $\boldsymbol{C}, \boldsymbol{D}$, Normalized and color-coded PSTHs aligned to the middle of the reward consumption for core $(\boldsymbol{C})$ and shell $(\boldsymbol{D})$. Consumptionexcited neurons are displayed at the top and consumption-inhibited neurons at the bottom. Within each category, neurons are sorted by response durations (shorter responses at the top for excitations and at the bottom for inhibitions). $\boldsymbol{E}, \boldsymbol{F}$, Average response for consumptionresponsive neurons in the core $(\boldsymbol{E})$ and shell $(\boldsymbol{F}) . \boldsymbol{G}, \mathrm{AUC}$ of consumption responses. ${ }^{*} p<0.05$, ${ }^{* * *} p<0.01$, compared to core.

(Fig. 8A). Inhibitions in the NAc core during reward consumption were far more frequent than in the shell (permutation test, $p<0.001$ ) (Fig. 8A,C,D). Although inhibitions in the core did not last significantly longer than in the shell (Fig. $8 B-D$ ) (twoway ANOVA structure $F_{(1,325)}=2.2, p=0.14$, response type $F_{(1,325)}=3.2, p=0.07$, structure $\times$ response type $F_{(1,325)}=2.4$, $p=0.12$ ), they had a larger AUC $\left(t_{(234)}=2.3, p=0.02\right)$ (Fig. $8 E-G)$. These strong inhibitions in the core are reminiscent of the long-lasting inhibitions found after DS presentation (Figs. $5 B, C, 6$ ).
A
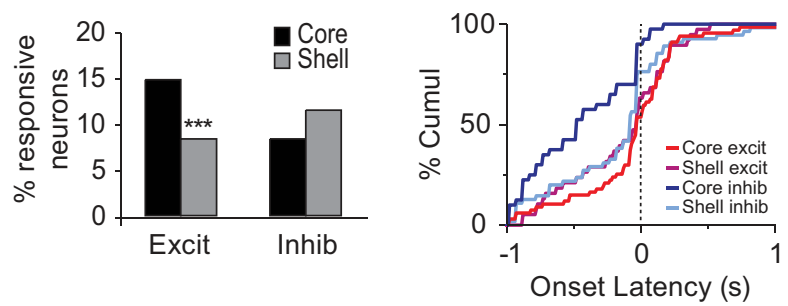

C Core
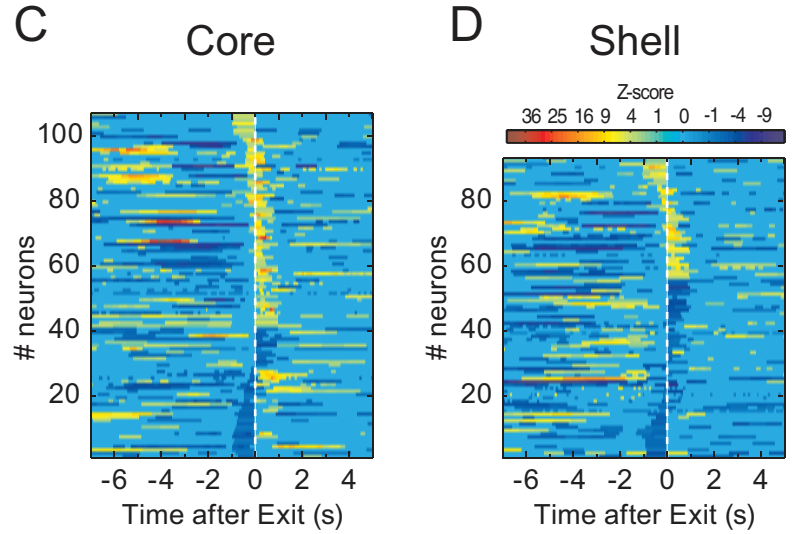

E
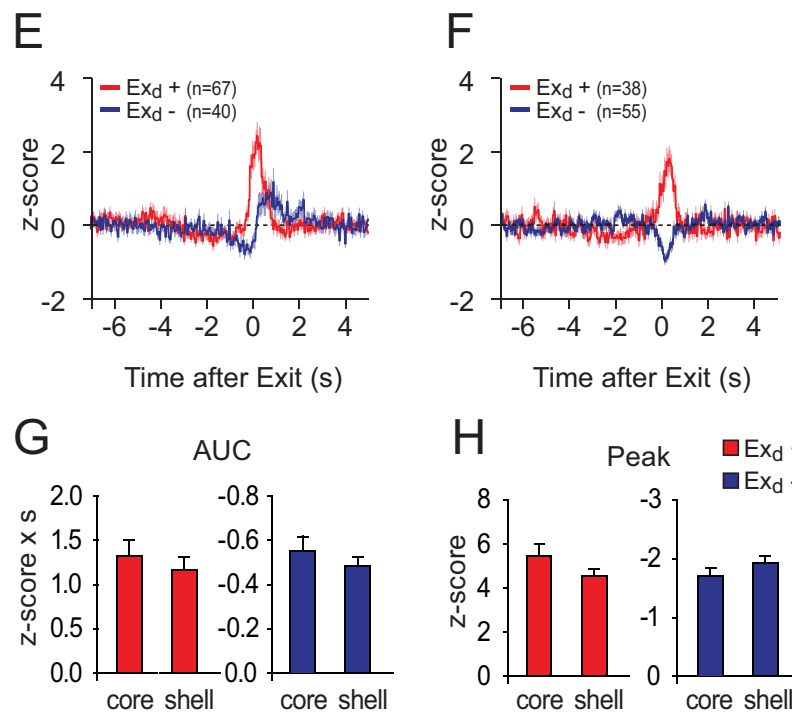

Figure 9. Neuronal responses to reward receptacle exit. $\boldsymbol{A}$, Percentage of excitation and inhibition in response to the reward receptacle exit in the core and the shell. $\boldsymbol{B}$, Cumulative percentage of onset latency of reward receptacle exit-related responses. $\boldsymbol{C}, \boldsymbol{D}$, Normalized and color-coded PSTHs aligned to the middle of the reward receptacle exit for core $(\boldsymbol{C})$ and shell (D). Reward receptacle exit-excited neurons are displayed at the top and reward receptacle exitinhibited neurons at the bottom. Within each category, neurons are sorted by response durations (shorter responses at the top for excitations and at the bottom for inhibitions). $\boldsymbol{E}, \boldsymbol{F}$, Average receptacle-exit responses for core $(\boldsymbol{E})$ and shell $(\boldsymbol{F})$ neurons. $\mathbf{G}, \boldsymbol{H}$, Peak $(\boldsymbol{G})$ and AUC $(\boldsymbol{H})$ of receptacle-exit responses. ${ }^{* * *} p<0.01$ compared to core.

Excitations in the NAc core displayed a trend toward a significantly greater firing rate increase relative to the shell $\left(t_{(91)}=1.8, p=0.07\right)$.

NAc responses to reward receptacle exit

Consistent with previous reports (Nicola et al., 2004a), NAc neurons respond to the exit of the reward receptacle. NAc core neurons were more often excited by receptacle exit (permutation test, $p<0.05$ ) (Fig. 9A,C,D). Response onset latency depended on the structure and the response direction (Fig. 
$9 B-D)$ (two-way ANOVA structure $F_{(1,194)}=2.9, p=0.09$, response type $F_{(1,194)}=13.8, p<0.001$, structure $\times$ response type $\left.F_{(1,194)}=8, p=0.005\right)$. Inhibition onset in the NAc core occurred earlier than onset of all other response types (Fig. 9B) (Newman-Keuls $p<0.05$ ). We found no difference between NAc core and shell neurons in term of response magnitude (AUC excitation, $t_{(102)}=0.58, p=0.56$; peak excitation, $t_{(102)}=1.29 p=$ 0.2 ; AUC inhibition, $t_{(92)}=-0.9, p=0.36$; peak inhibition, $t_{(92)}=$ $1.23, p=0.22$ ).

\section{Discussion}

Here we dissociate the effect of NAc core and shell inactivation in a discriminative stimulus task. Blockade of glutamate receptors in the core but not the shell impairs responding to a rewardpredictive cue. On the other hand, shell but not core inactivation produces a temporally specific increase in responding to the unrewarded cue. Although we did not find neuronal firing patterns unique to core or shell, we did find stronger neuronal representation of the DS in the core, and more selective representation of the NS in the shell. This is consistent with the hypothesis that DS responses in core promote behavioral responding to rewardpredictive cues, whereas NS responses in the shell suppress responding to less relevant cues.

\section{Role of the NAc core and shell in incentive-cue responding}

Inactivation or lesions of the NAc core impairs performance in tasks where discrete sensory cues control operant behaviors (Hall et al., 2001; Fuchs et al., 2004; Ito et al., 2004; Di Ciano et al., 2008; Floresco et al., 2008). However, inactivation of the entire NAc failed to decrease correct responding in the DS task and instead increased nonspecific responding (Yun et al., 2004a). The present study resolves this issue by showing that selectively inactivating the core reduces responding to the DS. Shell inactivation, on the other hand, strongly increases unrewarded responding. That general NAc inactivation failed to reduce DS-cued responding (Yun et al., 2004a) might therefore be due to a balance of opposing inhibitory and disinhibitory effects of combined core and shell inactivation on DS responding.

That core inactivation reduces DS responding suggests that neuronal activity occurring between the DS and the lever presses promotes responding. In line with this, NAc neuronal responses to the DS were greater when the rat made an operant response to the cue than when it did not (Nicola et al., 2004b). Overall, we found that both DS and lever-press neuronal responses were greater in core than shell. This may explain the differential involvement of the core and shell in promoting behavioral responding to the DS. On the other hand, many shell neurons also respond to the DS and lever press with characteristics similar to core neuron responses. Despite this, inactivating the shell had no effect on DS responding. Therefore, the differential contribution of core and shell to DS responding may have more to do with differences in the projection targets of neurons with similar firing patterns. For instance, the NAc core projects to the lateral ventral pallidum, the subthalamic nucleus, and the medial substantia nigra pars reticulata, while the shell projects to the medial ventral pallidum, VTA, and hypothalamus (Heimer et al., 1991; Groenewegen et al., 1999; Tripathi et al., 2010).

The similarity of DS encoding in NAc core and shell neurons raises the question of the origin of the afferent input that drives the DS responses in these subregions. We previously showed that the VTA is necessary for DS-evoked responses in NAc neurons (Yun et al., 2004b). Furthermore, cue-evoked dopamine release is similar in the core and shell (Wanat et al., 2010), consistent with the idea that dopamine promotes DS-evoked firing of both core and shell neurons. The BLA is also critical for DS firing in NAc neurons. BLA inactivation blocks DS-evoked firing in the core (Ambroggi et al., 2008; Jones et al., 2010). Although lesions of BLA reportedly failed to block shell DS responses (Jones et al., 2010), the rostral BLA, known to project preferentially to the core (Shinonaga et al., 1994), was targeted by Jones et al. (2010). The caudal BLA, which projects to the shell, could therefore contribute to cue-evoked responses in the shell. It is also possible that other distinct inputs contribute differentially to DS-driven responses in the core and shell. Indeed, the dorsal medial PFC, which projects preferentially to the core, promotes both behavioral and neuronal responses to incentive cues (Ishikawa et al., 2008 b). Whether shell responses are specific to other PFC inputs requires further investigation.

\section{Role of the NAc core and shell in suppressing task-irrelevant behaviors}

We found that NAc inactivation induced two different forms of behavioral disinhibition. One, produced by both core and shell inactivation, is a general increase in responding on both active and inactive levers. Previous studies found that such effects were specific to shell inactivation (Floresco et al., 2008; Peters et al., 2008). The larger volume injected in our study and possible diffusion from core to shell could explain this discrepancy. However, we also found a shell-specific form of behavioral disinhibition: a temporally specific increase in active lever pressing during the NS.

Our neuronal recordings cannot reveal potential physiological correlates of the nonspecific disinhibition of lever pressing caused by NAc inactivation. Because this behavior does not occur when the NAc is intact and has no specific temporal marker, it is impossible to correlate neuronal firing with successful inhibition of the behavior. However, more neurons with lever-press inhibitions are observed in shell than core. If activity in these neurons inhibits (and their inhibition permits) lever pressing, lever-press disinhibition would be, as we observed, stronger after shell inactivation. On the other hand, the increased responding during the NS window following shell inactivation indicates that NAc neurons can also generate a temporally specific response suppression during the NS. In fact, we found a greater population of neurons excited by the NS (but not by the DS) in the shell than in the core. Some of these shell neurons with NS-specific responses may inhibit operant responding promoted by the NS.

The population of NS responsive neurons in the shell was relatively small, as was the increase in NS-induced lever pressing following shell inactivation. What could explain this relatively weak effect? Rats initially lever pressed to the NS when it was first introduced in training (unpublished observations), and they had to learn to actively suppress such responding. Across several months of training, the degree of suppression of prepotent responding to the NS decreased over time. If shell neurons suppress responding, their involvement might be stronger at the beginning of extinction, when behavioral suppression is strongest. This hypothesis is supported by data obtained by Ghitza et al. (2003): NAc neurons were recorded on the first day of extinction of a similar discriminative stimulus task where the cue was presented but the reward (cocaine) was not. They report that shell neurons responded more strongly to the cue than those in core. We suggest that a population of shell neurons are recruited early in extinction and that these neurons actively suppress responding to a cue that is no longer associated with the reward. Interestingly, the ventral PFC, which preferentially targets the shell (Voorn et al., 
2004), has been implicated in extinction (Peters et al., 2008), and its inactivation induces behavioral disinhibition in the DS task (Ishikawa et al., 2008a). Therefore, activity related to action suppression in shell neurons may depend on ventral PFC inputs. Further experiments are needed to test this hypothesis.

\section{Role of excitations versus inhibitions in promoting and stopping behavior}

A critical unanswered question is whether excitation or inhibition of NAc neurons promotes or suppresses behavior. Because initial studies in primates did not report inhibitions in the striatum (Bowman et al., 1996; Hassani et al., 2001; Cromwell and Schultz, 2003), most of the theories of the function of the ventral and dorsal striatum are related to excitations (Hikosaka, 2007; Nicola, 2007; Humphries and Prescott, 2010). However, studies from this laboratory in rodents have shown that NAc neuronal inhibitions are prevalent in several appetitive behaviors (Nicola et al., 2004a; Taha and Fields, 2006; Krause et al., 2010). The long-lasting inhibitions, such as those reported here in the core, have been proposed to permit (gate) goal-directed behaviors. This hypothesis states that a subpopulation of NAc neurons tonically suppresses behavior and that their inhibition is necessary for a behavior to occur (Taha and Fields, 2006; Krause et al., 2010). The fact that these inhibitions are more widespread and longer lasting in the core might appear to contradict the stronger behavioral suppression exerted by the shell in this and other paradigms (Kelley and Swanson, 1997; Stratford and Kelley, 1997; Peciña and Berridge, 2000; Reynolds and Berridge, 2002). However, electrical stimulation of both NAc core and shell produces powerful inhibition of sucrose licking (Krause et al., 2010). Furthermore, inhibitions (or excitations) in the core and shell could have opposing effects on behavior. Selective manipulation of excited and inhibited neurons will be required to determine their specific behavioral roles.

\section{Conclusion}

The NAc core and shell make distinct contributions to the DS task. Our recordings revealed that while the two regions differed in the proportions of neurons with different firing patterns, which could partly explain their different contributions, no phasic event-related firing pattern was unique to either core or shell neurons. This raises the possibility that neurons with similar firing patterns in core and shell have different behavioral actions that depend on their distinct projection targets.

\section{References}

Ambroggi F, Ishikawa A, Fields HL, Nicola SM (2008) Basolateral amygdala neurons facilitate reward-seeking behavior by exciting nucleus accumbens neurons. Neuron 59:648-661.

Bowman EM, Aigner TG, Richmond BJ (1996) Neural signals in the monkey ventral striatum related to motivation for juice and cocaine rewards. J Neurophysiol 75:1061-1073.

Cardinal RN, Parkinson JA, Hall J, Everitt BJ (2002) Emotion and motivation: the role of the amygdala, ventral striatum, and prefrontal cortex. Neurosci Biobehav Rev 26:321-352.

Chaudhri N, Sahuque LL, Schairer WW, Janak PH (2010) Separable roles of the nucleus accumbens core and shell in context- and cue-induced alcohol-seeking. Neuropsychopharmacology 35:783-791.

Cromwell HC, Schultz W (2003) Effects of expectations for different reward magnitudes on neuronal activity in primate striatum. J Neurophysiol 89:2823-2838.

Di Ciano P, Everitt BJ (2001) Dissociable effects of antagonism of NMDA and AMPA/KA receptors in the nucleus accumbens core and shell on cocaine-seeking behavior. Neuropsychopharmacology 25:341-360.

Di Ciano P, Cardinal RN, Cowell RA, Little SJ, Everitt BJ (2001) Differential involvement of NMDA, AMPA/kainate, and dopamine receptors in the nucleus accumbens core in the acquisition and performance of pavlovian approach behavior. J Neurosci 21:9471-9477.

Di Ciano P, Robbins TW, Everitt BJ (2008) Differential effects of nucleus accumbens core, shell, or dorsal striatal inactivations on the persistence, reacquisition, or reinstatement of responding for a drug-paired conditioned reinforcer. Neuropsychopharmacology 33:1413-1425.

Fields HL, Hjelmstad GO, Margolis EB, Nicola SM (2007) Ventral tegmental area neurons in learned appetitive behavior and positive reinforcement. Annu Rev Neurosci 30:289-316.

Floresco SB, McLaughlin RJ, Haluk DM (2008) Opposing roles for the nucleus accumbens core and shell in cue-induced reinstatement of foodseeking behavior. Neuroscience 154:877-884.

Fuchs RA, Evans KA, Parker MC, See RE (2004) Differential involvement of the core and shell subregions of the nucleus accumbens in conditioned cue-induced reinstatement of cocaine seeking in rats. Psychopharmacology (Berl) 176:459-465.

Ghazizadeh A, Fields HL, Ambroggi F (2010) Isolating event-related neuronal responses by deconvolution. J Neurophysiol 104:1790-1802.

Ghitza UE, Fabbricatore AT, Prokopenko V, Pawlak AP, West MO (2003) Persistent cue-evoked activity of accumbens neurons after prolonged abstinence from self-administered cocaine. J Neurosci 23:7239-7245.

Groenewegen HJ, Wright CI, Beijer AV, Voorn P (1999) Convergence and segregation of ventral striatal inputs and outputs. Ann N Y Acad Sci 877:49-63.

Hall J, Parkinson JA, Connor TM, Dickinson A, Everitt BJ (2001) Involvement of the central nucleus of the amygdala and nucleus accumbens core in mediating Pavlovian influences on instrumental behaviour. Eur J Neurosci 13:1984-1992.

Hassani OK, Cromwell HC, Schultz W (2001) Influence of expectation of different rewards on behavior-related neuronal activity in the striatum. J Neurophysiol 85:2477-2489.

Heimer L, Zahm DS, Churchill L, Kalivas PW, Wohltmann C (1991) Specificity in the projection patterns of accumbal core and shell in the rat. Neuroscience 41:89-125.

Hikosaka O (2007) GABAergic output of the basal ganglia. Prog Brain Res 160:209-226.

Horne AL, Woodruff GN, Kemp JA (1990) Synaptic potentials mediated by excitatory amino acid receptors in the nucleus accumbens of the rat, in vitro. Neuropharmacology 29:917-921.

Humphries MD, Prescott TJ (2010) The ventral basal ganglia, a selection mechanism at the crossroads of space, strategy, and reward. Prog Neurobiol 90:385-417.

Ishikawa A, Ambroggi F, Nicola SM, Fields HL (2008a) Contributions of the amygdala and medial prefrontal cortex to incentive cue responding. Neuroscience 155:573-584.

Ishikawa A, Ambroggi F, Nicola SM, Fields HL (2008b) Dorsomedial prefrontal cortex contribution to behavioral and nucleus accumbens neuronal responses to incentive cues. J Neurosci 28:5088-5098.

Ito M, Doya K (2009) Validation of decision-making models and analysis of decision variables in the rat basal ganglia. J Neurosci 29:9861-9874.

Ito R, Robbins TW, Everitt BJ (2004) Differential control over cocaineseeking behavior by nucleus accumbens core and shell. Nat Neurosci 7:389-397.

Jones JL, Day JJ, Wheeler RA, Carelli RM (2010) The basolateral amygdala differentially regulates conditioned neural responses within the nucleus accumbens core and shell. Neuroscience 169:1186-1198.

Kelley AE, Swanson CJ (1997) Feeding induced by blockade of AMPA and kainate receptors within the ventral striatum: a microinfusion mapping study. Behav Brain Res 89:107-113.

Krause M, German PW, Taha SA, Fields HL (2010) A pause in nucleus accumbens neuron firing is required to initiate and maintain feeding. J Neurosci 30:4746-4756.

Nicola SM (2007) The nucleus accumbens as part of a basal ganglia action selection circuit. Psychopharmacology (Berl) 191:521-550.

Nicola SM (2010) The flexible approach hypothesis: unification of effort and cue-responding hypotheses for the role of nucleus accumbens dopamine in the activation of reward-seeking behavior. J Neurosci 30: $16585-16600$

Nicola SM, Kombian SB, Malenka RC (1996) Psychostimulants depress excitatory synaptic transmission in the nucleus accumbens via presynaptic D1-like dopamine receptors. J Neurosci 16:1591-1604.

Nicola SM, Surmeier J, Malenka RC (2000) Dopaminergic modulation of 
neuronal excitability in the striatum and nucleus accumbens. Annu Rev Neurosci 23:185-215.

Nicola SM, Yun IA, Wakabayashi KT, Fields HL (2004a) Firing of nucleus accumbens neurons during the consummatory phase of a discriminative stimulus task depends on previous reward predictive cues. J Neurophysiol 91:1866-1882.

Nicola SM, Yun IA, Wakabayashi KT, Fields HL (2004b) Cue-evoked firing of nucleus accumbens neurons encodes motivational significance during a discriminative stimulus task. J Neurophysiol 91:1840-1865.

Peciña S, Berridge KC (2000) Opioid site in nucleus accumbens shell mediates eating and hedonic 'liking' for food: map based on microinjection Fos plumes. Brain Res 863:71-86.

Pennartz CM, Boeijinga PH, Kitai ST, Lopes da Silva FH (1991) Contribution of NMDA receptors to postsynaptic potentials and paired-pulse facilitation in identified neurons of the rat nucleus accumbens in vitro. Exp Brain Res 86:190-198.

Peters J, LaLumiere RT, Kalivas PW (2008) Infralimbic prefrontal cortex is responsible for inhibiting cocaine seeking in extinguished rats. J Neurosci 28:6046-6053.

Reynolds SM, Berridge KC (2002) Positive and negative motivation in nucleus accumbens shell: bivalent rostrocaudal gradients for GABA-elicited eating, taste "liking"/“disliking" reactions, place preference/avoidance, and fear. J Neurosci 22:7308-7320.

Roesch MR, Singh T, Brown PL, Mullins SE, Schoenbaum G (2009) Ventral striatal neurons encode the value of the chosen action in rats deciding between differently delayed or sized rewards. J Neurosci 29:13365-13376.

Roitman MF, Wheeler RA, Carelli RM (2005) Nucleus accumbens neurons are innately tuned for rewarding and aversive taste stimuli, encode their predictors, and are linked to motor output. Neuron 45:587-597.

Shinonaga Y, Takada M, Mizuno N (1994) Topographic organization of collateral projections from the basolateral amygdaloid nucleus to both the prefrontal cortex and nucleus accumbens in the rat. Neuroscience 58:389-397.

Stratford TR, Kelley AE (1997) GABA in the nucleus accumbens shell participates in the central regulation of feeding behavior. J Neurosci 17: $4434-4440$

Taha SA, Fields HL (2005) Encoding of palatability and appetitive behaviors by distinct neuronal populations in the nucleus accumbens. J Neurosci 25:1193-1202.

Taha SA, Fields HL (2006) Inhibitions of nucleus accumbens neurons encode a gating signal for reward-directed behavior. J Neurosci 26:217-222.

Taha SA, Nicola SM, Fields HL (2007) Cue-evoked encoding of movement planning and execution in the rat nucleus accumbens. J Physiol 584: 801-818.

Tripathi A, Prensa L, Cebrián C, Mengual E (2010) Axonal branching patterns of nucleus accumbens neurons in the rat. J Comp Neurol 518: 4649-4673.

Voorn P, Vanderschuren LJ, Groenewegen HJ, Robbins TW, Pennartz CM (2004) Putting a spin on the dorsal-ventral divide of the striatum. Trends Neurosci 27:468-474.

Wanat MJ, Kuhnen CM, Phillips PE (2010) Delays conferred by escalating costs modulate dopamine release to rewards but not their predictors. J Neurosci 30:12020-12027.

Yun IA, Nicola SM, Fields HL (2004a) Contrasting effects of dopamine and glutamate receptor antagonist injection in the nucleus accumbens suggest a neural mechanism underlying cue-evoked goal-directed behavior. Eur J Neurosci 20:249-263.

Yun IA, Wakabayashi KT, Fields HL, Nicola SM (2004b) The ventral tegmental area is required for the behavioral and nucleus accumbens neuronal firing responses to incentive cues. J Neurosci 24:2923-2933. 